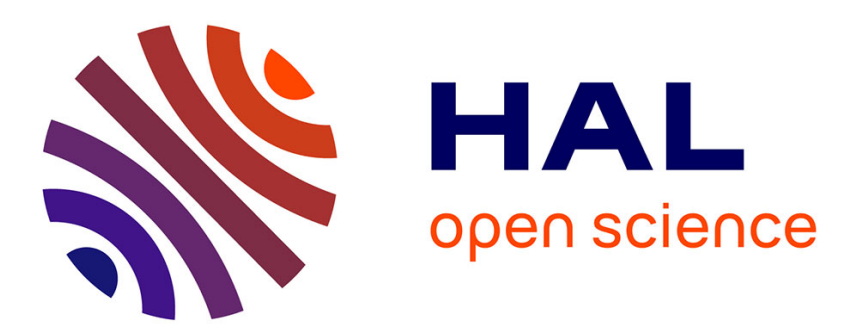

\title{
How does imposing free roaming in EU impact users and ISPs' relations?
}

\author{
Patrick Maillé, Bruno Tuffin
}

\section{To cite this version:}

Patrick Maillé, Bruno Tuffin. How does imposing free roaming in EU impact users and ISPs' relations?. 8th International Conference Network of the Future, Nov 2017, London, United Kingdom. hal01493911

\section{HAL Id: hal-01493911 \\ https://hal.inria.fr/hal-01493911}

Submitted on 29 Mar 2017

HAL is a multi-disciplinary open access archive for the deposit and dissemination of scientific research documents, whether they are published or not. The documents may come from teaching and research institutions in France or abroad, or from public or private research centers.
L'archive ouverte pluridisciplinaire $\mathbf{H A L}$, est destinée au dépôt et à la diffusion de documents scientifiques de niveau recherche, publiés ou non, émanant des établissements d'enseignement et de recherche français ou étrangers, des laboratoires publics ou privés. 


\title{
How does imposing free roaming in EU impact users and ISPs' relations?
}

\author{
Patrick Maillé \\ IMT Atlantique \\ Bruno Tuffin \\ Rennes, France \\ Inria \\ Email: patrick.maille@imt.fr Email: bruno.tuffin@inria.fr
}

\begin{abstract}
Starting in June 2017, roaming within Europe will become free for mobile phone users, as an enforcement of the European parliament. The goal being to obtain a unified digital market: this seems at first sight to largely benefit to users, who will not have to worry about their usage abroad. We design in this paper a model analyzing the impact of free roaming enforcement on users and ISPs' pricing strategies, with respect to paid roaming to verify this assumption. We particularly focus on the strategies on transit payments between ISPs in different countries. We highlight that scrutiny is required since, depending on parameters, consumer surplus or subscription penetration are not necessarily maximized if free roaming is enforced.
\end{abstract}

\section{Introduction}

In traditional mobile network charging plans, roaming in a foreign country implies additional charges, typically volume based or cap based. But in October 2015 the European parliament has decided to forbid roaming charges, starting June 15, 2017, in order to have a unified digital market ${ }^{1}$. Users then just need to subscribe to a plan in their member state, determined as the place where they spend most of their time. As a transitory phase, the roaming charges have already been capped since April 2016.

This free roaming rule does seem attractive at first sight, with mobile users not having to worry about their data consumption while travelling all over Europe. But it may lead to negative effects that we wish to investigate here: i) Despite being appealing, is there a risk to create a price increase by which "poor" domestic-only users would pay for "rich" roaming users? ii) What is the impact of free roaming on transit prices between ISPs in different countries, and then on subscription prices? iii) Is it needed to regulate the way transit

${ }^{1}$ https://ec.europa.eu/digital-single-market/en/news/new-rules-roaming-charges-and-open-internet 
prices are decided, or can we leave it open to ISPs (through negotiation or a non-cooperative game)?

In order to answer those questions, we design a model representing the relations between ISPs in two different countries. Each ISP decides the flat rate price for its domestic users and the transit price (for traffic from the ISP in the foreign country) charged to the counterpart ISP. To make comparisons with the case of paid roaming, we will also add the possibility of a volume-based fee for roaming usage, charged to its own users for data consumption abroad.

The two cases, free and paid roaming will be analyzed thanks to noncooperative game theory $[2,6]$, since the decision of an ISP will impact the output (the revenue) of the distant ISP from the transit fee and the number of roamers. We will show that scrutiny is required since some a priori counterintuitive results come out. We will illustrate among other things that consumers do not always benefit from free roaming: consumer surplus, representing the aggregated net benefit from users, might even be larger with paid roaming.Due to the effect of prices on subscription rates, free roaming might also lead to less subscriptions.

To our knowledge, a very limited amount of research work exists on the analysis of the enforcement of free roaming, and even on roaming in general. We can cite [7], but it is rather focusing on national roaming of customers and cooperation among operators. Recently, we have designed and analyzed in [4] a model looking at a single ISP optimizing its pricing strategies, both in case of paid and free roaming, and comparing them. Our model makes use of the same representation of users' preferences, but extends it in the sense that it additionally investigates the impact of relations between ISPs in the different countries based on the negotiated transit prices, which can influence the domestic decisions and induce a new level of decisions.

The remainder of this paper is organized as follows. Section 2 presents our model of user preferences and operator revenues as well as the different levels of decisions. In Section 3, we recall the results on users' choices in terms of subscription and data usage depending on prices, as obtained in [4]. Section 4 provides some theoretical results on the users' pricing game between ISPs, while Section 5 analyzes transit prices being decided either from a non-cooperative game, through a negotiation (using the so-called Nash bargaining solution [6]), or by a regulator in order to maximize consumer surplus. Section 6 then compares the outcomes of the combinations of scenarios to determine what would be the best strategy for all actors and discusses the UE parliament decision. Section 7 particularly focuses on the impact of decisions on each individual of the population for an arbitrarily chosen scenario. Finally, we conclude in Section 8. 


\section{Model}

We consider two countries for simplicity, differentiated by indices 1 and 2, but more countries could be considered as well. According to the rules/recommendations ${ }^{2}$, citizens of a country have to subscribe to the/an ISP in their own country to avoid an unfair competition due to different local constraints. We thus avoid the possibility of a citizen of a given country subscribing in another and less expensive country and consuming freely in its own country.

\subsection{Users' representation}

In each Country $i \in\{1,2\}$, users have heterogeneous willingness-to-pay values (in monetary units per month) for a flat-rate domestic subscription; we denote that value for a particular user by $\theta_{i}$, and assume that the distribution of $\theta_{i}$ over the market is known to the operator. The cumulative distribution function of $\theta_{i}$ is denoted by $F_{i}$ and will help us to represent two countries with different GDP per capita, a situation for which free roaming could have a more important impact. In all our numerical analysis, we will assume that $\theta_{i}$ is exponentially distributed with rate $\lambda_{i}$. Then the smaller $\lambda_{i}$, the larger the average wealth. There is a total mass (population) $m_{i}$ of potential users in Country $i$.

But users are not only interested in a domestic usage of their mobile phone, as they can in addition be willing to consume data while traveling abroad (that is, while roaming). Here too there is some heterogeneity in the willingnessto-pay for data while roaming, but it is reasonable to assume a positive correlation between the willingness-to-pay $\theta_{i}$ for the domestic data plan, and the willingness-to-pay function (in terms of volume) for roaming data. Let us denote this willingness-to-pay for roaming data by $r_{\theta_{i}}(x)$ for a consumed volume $x$ over a month. All over the paper, we are going to consider the commonly adopted following form of $r_{\theta_{i}}$, see [1,3]:

Assumption A For any value of $\theta$, the function $r_{\theta}$ is such that

$$
r_{\theta}(x)= \begin{cases}\theta x-\alpha \frac{x^{2}}{2} & \text { if } x \leq \frac{\theta}{\alpha} \\ \frac{\theta^{2}}{2 \alpha} & \text { otherwise. }\end{cases}
$$

with $\alpha$ a fixed parameter. The marginal valuation function $r_{\theta}^{\prime}$ then has the simple expression

$$
r_{\theta}^{\prime}(x)=[\theta-\alpha x]^{+}
$$

where $[y]^{+}:=\max (y, 0)$.

This specific form verifies expected properties: for any value of $\theta_{i}$, it is nonnegative, null at 0 , non-decreasing, concave and bounded. Moreover, it verifies that users with high willingness-to-pay $\theta_{i}$ for domestic usage are also willing to

\footnotetext{
${ }^{2}$ see again https://ec.europa.eu/digital-single-market/en/news/ new-rules-roaming-charges-and-open-internet
} 
pay more for roaming data usage than users with low $\theta_{i}$ (the so-called "positive correlation" mentioned above).

The ISP in Country $i$ is charging a flat-rate $p_{i}$ to its subscribers for a domestic usage. We will compare free and paid roaming, so let us define $c_{i}$ as the fixed per-volume price for roaming data; free roaming will just mean $c_{i}=0$.

Given those notations, the net utility of a user with domestic willingness-topay $\theta_{i}$ in Country $i$ and consuming a volume $v_{i}\left(\theta_{i}\right)$ of roaming data per month (on average), is

$$
U_{i}\left(\theta_{i}\right):=\theta_{i}-p_{i}+r_{\theta_{i}}\left(v_{i}\left(\theta_{i}\right)\right)-c_{i} v_{i}\left(\theta_{i}\right),
$$

where $\theta_{i}-p_{i}$ stands for the net utility for domestic usage and $r_{\theta_{i}}\left(v_{i}\left(\theta_{i}\right)\right)-c_{i} v_{i}\left(\theta_{i}\right)$ is the net utility for roaming. That form defines the behavior of any user, subscribing if getting a non-negative net utility. Let

$$
D_{i}:=\left\{\theta_{i}: U_{i}\left(\theta_{i}\right) \geq 0\right\}
$$

be the set of users $\theta_{i}$ subscribing to the offer of ISP $i$, meaning that its associated demand-the mass of subscribers to ISP $i$-is

$$
d_{i}:=m_{i} \int_{D_{i}} \mathrm{~d} F_{i}\left(\theta_{i}\right) .
$$

\subsection{ISPs' revenues and Consumer Surplus}

Each ISP $i$ chooses its subscription price $p_{i}$, and (in case of paid roaming) the roaming volume-based price $c_{i}$ charged to users, but can also charge a transit price $t_{i}$ to the counterpart ISP for the traffic from foreign users using its network. There are thus three decision variables for each ISP. We here potentially consider that an ISP may charge more its own users than what it has to pay itself for roaming data, that is, $c_{i}$ can be larger than $t_{j}$ with $j \neq i$.

From those assumptions, the revenue (that is, the utility) that ISP $i$ tries to maximize is

$$
R_{i}=d_{i} p_{i}+\left(c_{i}-t_{j}\right) m_{i} \int_{D_{i}} v_{i}\left(\theta_{i}\right) \mathrm{d} F_{i}\left(\theta_{i}\right)+t_{i} m_{j} \int_{D_{j}} v_{j}\left(\theta_{j}\right) \mathrm{d} F_{j}\left(\theta_{j}\right) .
$$

Another measure of interest, especially from a European regulator (the BEREC) point of view seeking to maximize user welfare, is the consumer surplus representing the aggregated net utility of users over all countries:

$$
\mathrm{CS}=\sum_{i} m_{i} \int\left[U_{i}\left(\theta_{i}\right)\right]^{+} \mathrm{d} F_{i}\left(\theta_{i}\right)=\sum_{i} m_{i} \int_{D_{i}} U_{i}\left(\theta_{i}\right) \mathrm{d} F_{i}\left(\theta_{i}\right) .
$$

\subsection{Scenarios and hierarchy of decisions}

Several scenarios can be considered for the decision of transit prices $t_{1}$ and $t_{2}$, while prices charges to users are always chosen to maximize the ISPs' revenues. 
In all cases, we will compare the free roaming situation where $c_{1}=c_{2}=0$ with the paid roaming.

The scenarios for the decision on the transit prices are the following:

1. $t_{1}$ and $t_{2}$ decided by a regulator to maximize the consumer surplus;

2. $t_{1}$ and $t_{2}$ decided as a Nash Bargaining solution [6] under the threat that the regulator will choose of no agreement is found;

3. $\left(t_{1}, t_{2}\right)$ a Nash equilibrium as the result of a non-cooperative game.

There is a natural order of decisions:

1. The transit price(s) $t_{i} \forall i$ are first decided according to one of the three above possibilities;

2. Then prices are defined by the ISPs: $p_{i} \forall i$, as well as the $c_{i}$ S in case of paid roaming;

3. Based on all those values users decide to subscribe or not, defining the domains $D_{i} \forall i$.

But decisions are taken anticipatively, meaning that decisions at a given level will be made anticipating the decisions afterwards. The game is thus analyzed by so-called backward induction [2].

We therefore first compute the domains $D_{i}$ over $\theta_{i}$ of users choosing to subscribe in terms of all other parameters, then the ISP prices using those $D_{i} \mathrm{~s}$ for any choice of $t_{i} \mathrm{~s}$, and then the $t_{i} \mathrm{~s}$ using the anticipated (previously computed) values.

\section{User decisions}

Since the form of the user utility function described in Subsection 2.1 is taken from the literature, the derivations of user choices have already been studied. Based on utility function (1), it has been shown in [4] in the context of a single ISP and considering a single country, but the results remain valid for our model, that

- Under Assumption A, by a simple derivation with respect to $v_{i}$, the consumed volume is for a user $\theta_{i}$ is $v_{i}^{*}\left(\theta_{i}\right)=\left[\frac{\theta_{i}-c_{i}}{\alpha_{i}}\right]^{+}$, so the user subscribes if and only if $\theta+\frac{(\theta-c)^{2}}{2 \alpha} \mathbb{1}_{\{\theta>c\}}>p$.

- For any price pair $\left(p_{i}, c_{i}\right)$ the users who subscribe are those with $\theta_{i}>\bar{\theta}_{i}$, where

$$
\bar{\theta}_{i}=\min \left(p_{i}, c_{i}-\alpha_{i}+\sqrt{\alpha_{i}^{2}+2 \alpha_{i}\left[p_{i}-c_{i}\right]^{+}}\right),
$$

leading to

$$
D_{i}=\left(\bar{\theta}_{i}, \infty\right) .
$$

In words, users with valuations above the threshold $\bar{\theta}_{i}$ will subscribe. 


\section{On the pricing game between ISPs}

For fixed values of $t_{1}$ and $t_{2}$, what is the output of the pricing game between ISPs? In their choice, ISPs anticipate the reaction of users, hence know the values $\bar{\theta}_{1}$ and $\bar{\theta}_{2}$ for any profile of subscription (and roaming usage, if any) prices.

We have a non-cooperative game because the revenue (2) of one ISP depends on the prices of the other ISP through the demand for roaming data from this other ISP. However, for free and paid roaming, we have the following result which simplifies the analysis.

Proposition 1 The determination of revenue-optimizing prices $\left(p_{i}, c_{i}\right)$ of ISPs is independent of the pricing strategy $\left(p_{j}, c_{j}\right)$ of the other ISP $j$.

Proof: It actually comes from the expression (2) for the revenue of ISP $i$. The best response of ISP $i$ is obtained by differentiating (2) with respect to $p_{i}$ and $c_{i}$ (or just with respect to $p_{i}$ if $c_{i}=0$ in the case of free roaming). Revenue $R_{i}$ is made of three components:

- $d_{i} p_{i}$ which depends only on decision variables of ISP $i$;

- $\left(c_{i}-t_{j}\right) m_{i} \int_{D_{i}} v_{i}\left(\theta_{i}\right) \mathrm{d} F_{i}\left(\theta_{i}\right)$ which depends on ISP $j$ only through $t_{j}$, but that is a constant at this level of the game;

- $t_{i} m_{j} \int_{D_{j}} v_{j}\left(\theta_{j}\right) \mathrm{d} F_{j}\left(\theta_{j}\right)$ which does not depend on $\left(p_{i}, c_{i}\right)$, and therefore is a constant for the optimization at this level of the game.

As a consequence, we get the proposition.

For free roaming, we have the expression of revenue of ISP $i$

$$
R_{i}=d_{i} p_{i}-t_{j} m_{i} \int_{D_{i}} v_{i}\left(\theta_{i}\right) \mathrm{d} F_{i}\left(\theta_{i}\right)+t_{i} m_{j} \int_{D_{j}} v_{j}\left(\theta_{j}\right) \mathrm{d} F_{j}\left(\theta_{j}\right) .
$$

With exponential distributions for $\theta_{1}$ and $\theta_{2}$ and the expressions of $\bar{\theta}_{1}$ and $\bar{\theta}_{2}$ given in previous section, it gives for free roaming

$$
\begin{aligned}
R_{i}=m_{i} e^{-\lambda_{i} \bar{\theta}_{i}} p_{i} & -t_{j} \frac{m_{i}}{\alpha_{i}}\left(e^{-\lambda_{i} \bar{\theta}_{i}} \bar{\theta}_{i}+\frac{1}{\lambda_{i}} e^{-\lambda_{i} \bar{\theta}_{i}}\right) \\
& +t_{i} \frac{m_{j}}{\alpha_{j}}\left(e^{-\lambda_{j} \bar{\theta}_{j}} \bar{\theta}_{j}+\frac{1}{\lambda_{j}} e^{-\lambda_{j} \bar{\theta}_{j}}\right),
\end{aligned}
$$

and, for paid roaming,

$$
\begin{aligned}
R_{i}=m_{i} e^{-\lambda_{i} \bar{\theta}_{i}} p_{i} \quad- & \left(c_{i}-t_{j}\right) \frac{m_{i}}{\alpha_{i}}\left(e^{-\lambda_{i} \bar{\theta}_{i}}\left[\bar{\theta}_{i}-c_{i}\right]^{+}+\frac{e^{-\lambda_{i} c_{i}}}{\lambda_{i}} e^{-\lambda_{i}\left[\bar{\theta}_{i}-c_{i}\right]^{+}}\right) \\
& +t_{i} \frac{m_{j}}{\alpha_{j}}\left(e^{-\lambda_{j} \bar{\theta}_{j}}\left[\bar{\theta}_{j}-c_{j}\right]^{+}+\frac{e^{-\lambda_{j} c_{j}}}{\lambda_{j}} e^{-\lambda_{j}\left[\bar{\theta}_{j}-c_{j}\right]^{+}}\right) .
\end{aligned}
$$

Computing the revenue-maximizing prices analytically seems intractable, but it can easily be done numerically. For example when $\alpha_{1}=\alpha_{2}=1, \lambda_{1}=1$, $\lambda_{2}=1.5, m_{1}=1, m_{2}=2$ and $t_{1}=t_{2}=0.1$, we get as revenue-optimizing prices $\left(p_{1}, c_{1}\right)=(1,1.1)$ and $\left(p_{2}, c_{2}\right)=(0.67,0.765)$. 


\section{On the determination of transit prices}

Again, we are going to consider as much as possible paid and free roaming simultaneously, since free roaming can be summarized by taking $c_{1}=c_{2}=0$.

\subsection{Transit price decided by a regulator}

As a first possibility, we can let a regulator (like BEREC, the European one) decide the transit prices $t_{1}$ and $t_{2}$. A goal would be then to maximize the consumer surplus

$$
\begin{aligned}
\mathrm{CS} & =\sum_{i} m_{i} \int_{D_{i}} U_{i}\left(\theta_{i}\right) \mathrm{d} F_{i}\left(\theta_{i}\right) \\
& =\sum_{i} m_{i} \int_{D_{i}}\left(\theta_{i}-p_{i}+\left(\left[\theta_{i}-c_{i}\right]^{+}\right)^{2} /\left(2 \alpha_{i}\right)\right) \mathrm{d} F_{i}\left(\theta_{i}\right) .
\end{aligned}
$$

For free roaming, it reduces to $\mathrm{CS}=\sum_{i} m_{i} \int_{D_{i}}\left(\theta_{i}-p_{i}+\theta_{i}^{2} /\left(2 \alpha_{i}\right)\right) \mathrm{d} F_{i}\left(\theta_{i}\right)$.

Remark first that the expression of CS does not directly depend on the $t_{i} \mathrm{~s}$, but it does through the chosen $p_{i}$ s, which depend on the $t_{i}$ s from the second term in (2).

Through extensive computations, we have found in all our experiments that CS is maximized at $\left(t_{1}=0, t_{2}=0\right)$. We do not claim that it is always the case, but it seems to happen, at least with our parameters. Remark to understand what could drive to this result that a sufficient condition for CS to be maximized at $\left(t_{1}=0, t_{2}=0\right)$ would be that the $p_{i}$ s and $c_{i}$ s are non-decreasing with the $t_{i} \mathrm{~s}$ since $D_{i}$ then decreases. The increase of $p_{i}$ in terms of $t_{j}$ depends on the respective evolution of the derivatives (in terms of $p_{i}$ ) of $d_{i} p_{i}$ and $m_{i} \int_{D_{i}} v_{i}\left(\theta_{i}\right) \mathrm{d} F_{i}\left(\theta_{i}\right)$, but a proof or simple condition for seems hard to find and is out of the scope of this paper.

\subsection{Transit prices as a Nash bargaining solution}

Another solution is to consider the so-called Nash bargaining solution [5], output of a negotiation between ISPs: each ISP independently chooses a set of acceptable transit prices, and if the intersection of those sets is non-empty, the transit price is arbitrarily taken in the intersection. If ISPs do not agree with this, a threat is executed. A reasonable threat here is to take zero transit prices because if no agreement is found, the regulator can impose that no transit fee be applied. This negotiation scheme has in general several equilibria, but in [5] it is stated that the most likely outcome is the one maximizing the product of the utilities minus the utility at the threat situation. Formally, if $R_{i}\left(t_{1}, t_{2}\right)$ is the revenue if ISP $i$ where the roles of $t_{1}$ and $t_{2}$ are highlighted (with the corresponding equilibrium subscription fees), then the Nash bargaining solution $\left(t_{1}^{B}, t_{2}^{B}\right)$ is defined as:

$$
\left(t_{1}^{B}, t_{2}^{B}\right) \in \arg \max _{\left(t_{1}, t_{2}\right)}\left(R_{1}\left(t_{1}, t_{2}\right)-R_{1}(0,0)\right)\left(R_{2}\left(t_{1}, t_{2}\right)-R_{2}(0,0)\right),
$$




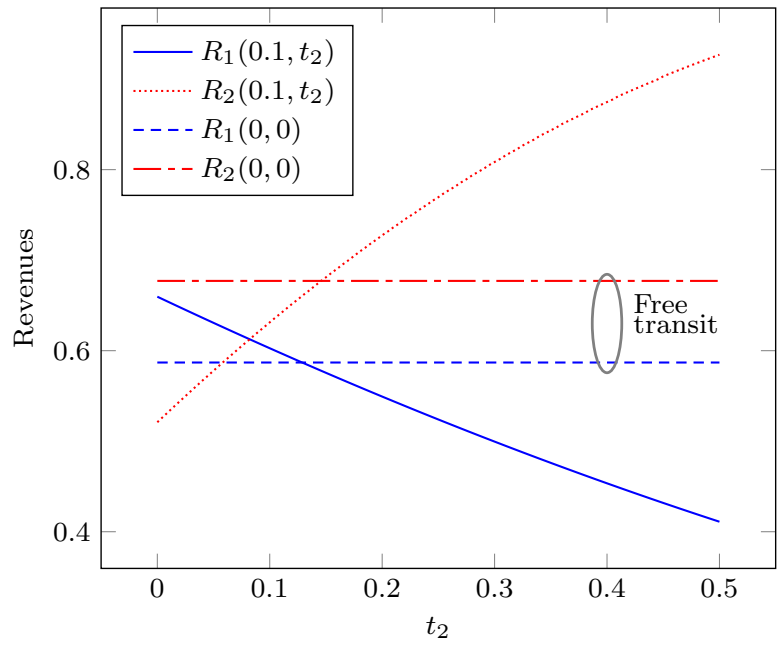

Figure 1: Free-roaming ISP revenues for $t_{1}=0.1$, with $\alpha_{1}=\alpha_{2}=1, \lambda_{1}=1$, $\lambda_{2}=1.5, m_{1}=1, m_{2}=2$. Subscription prices are set through a non-cooperative game among ISPs.

with the constraint that both terms are non-negative, because otherwise the ISPs prefer the no-transit-fee solution.

Again, during all our numerical investigations, the Nash bargaining solution was also at $(0,0)$. The reason is that with combinations of $\left(t_{1}, t_{2}\right)$ at least one of the ISPs get a smaller revenue than when it is $(0,0)$. We can illustrate this in Figure 1, where we plot for $t_{1}=0.1$ the revenues in terms of $t_{2}$ and the threat values $R_{1}(0,0), R_{2}(0,0)$, in the case of free roaming (that is, $c_{1}=c_{2}=0$ ).

\subsection{Transit prices from a game between ISPs}

Finally, the couple $\left(t_{1}, t_{2}\right)$ can be the output (a Nash equilibrium [6]) of a noncooperative game between ISPs, since the revenues depend on the transit price of the other ISP. But similarly to the users' pricing game, we have the following result saying that even if there is a dependence of revenues, the best-response transit price of an ISP does not depend on the strategy of the other ISP.

Proposition 2 The revenue-optimizing transit price $t_{i}$ of ISP $i$ is independent of the strategy $t_{j}$ of ISP $j$ (even if its revenue is not).

Proof: First remark that in the proof of Proposition 1, $p_{i}$ (and $c_{i}$ if paid roaming is considered), and therefore $D_{i}$ and $d_{i}$ depend on $t_{j}$ but not on $t_{i}$. Therefore, examining the expression (2) we observe that

- $d_{i} p_{i}+\left(c_{i}-t_{j}\right) m_{i} \int_{D_{i}} v_{i}\left(\theta_{i}\right) \mathrm{d} F_{i}\left(\theta_{i}\right)$ depends only on $t_{j}$ and not on $t_{i}$;

- $t_{i} m_{j} \int_{D_{j}} v_{j}\left(\theta_{j}\right) \mathrm{d} F_{j}\left(\theta_{j}\right)$ is the component that depends on $t_{i}$ and not on $t_{j}$ since $D_{j}$ depends on $t_{i}$ and not $t_{j}$. 
As a consequence, we get the proposition.

\section{Comparison and discussion}

In this section, we compare the outputs of the various scenarios, with transit prices chosen by the regulator, as a Nash bargaining solution or through a noncooperative game, each time for free and paid roaming.

As discussed above, transit prices chosen by the regulator or as a Nash bargaining solution always give $\left(t_{1}, t_{2}\right)=(0,0)$ and are therefore merged into one case.

In the first subsection, we discuss the output when varying $\lambda_{1}$, the other parameters being fixed. We also studied the variations in terms of other parameters but it did not provide any meaningful information; for example varying $m_{1}$ did not bring any significant impact on the various considered metrics. In the last subsection, we discuss for specific parameters the impact on each individual $\theta_{i}$ of the various strategies: free or paid roaming, combined with transit prices decided from a non-cooperative game or chosen as $(0,0)$.

\subsection{Impact of wealth parameter $\lambda_{1}$ in Country 1}

Let us fix (arbitrarily) the other model parameters, to $\alpha_{1}=\alpha_{2}=1, m_{1}=1$, $m_{2}=2$ and $\lambda_{2}=1$. We investigate the impact of $\lambda_{1}$ for the different strategies on CS in Figure 2, on $p_{i}$ in Figure 3, on $c_{i}$ (in the paid roaming cases) in Figure 4, on transit prices in Figure 5, on revenues of ISPs in Figure 6, on the thresholds $\bar{\theta}_{i}$ in Figure 7 , and on the proportions to subscribe $\bar{F}_{i}\left(\bar{\theta}_{i}\right)$ in each country in Figure 8.

Discussing the impact of the density of valuation in Country 1 on CS, we can remark that the larger $\lambda_{1}$, that is the smaller the valuations in distribution, then the smaller the CS at equilibrium. The situation with null transit prices (that is, decided by Nash bargaining or the regulator) and free roaming is (here) always giving the largest CS among all possibilities, hence should be the preferred situation for a regulator. Null transit prices always give a better CS that when transit prices are decided through a non-cooperative game, be it with free or paid roaming. Finally, focusing on paid and free roaming, when transit prices $\left(t_{1}, t_{2}\right)$ are chosen through a non-cooperative game, there is no clear better-performing scheme, in terms of consumer surplus, between free and paid roaming. It means that enforcing free roaming could actually be a false good idea depending on how prices are decided if transit price decisions are let to ISPs.

Looking at prices, wealth $\left(\lambda_{1}\right)$ in a country does not impact the pricing strategies $\left(p_{2}, c_{2}\right)$ in the other country. We can see on Figure 3 that the smaller $\lambda_{1}$ is, i.e., the larger the number of users with high valuation is, the larger the subscription price $p_{1}$ and roaming prices $c_{1}$ are, because users are more likely to accept high prices. Also free roaming induces a higher price because ISP 1 cannot get more money based on volume. Moreover subscription prices with free roaming are higher at Nash-equilibrium transit prices than with $(0,0)$ to 


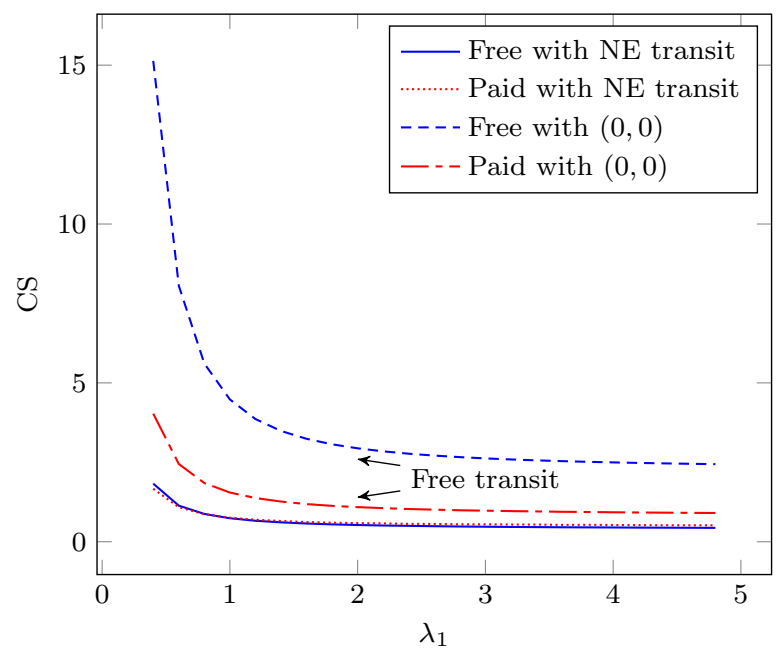

Figure 2: Consumer Surplus in terms of $\lambda_{1}$, for free and paid roaming, with different transit prices.

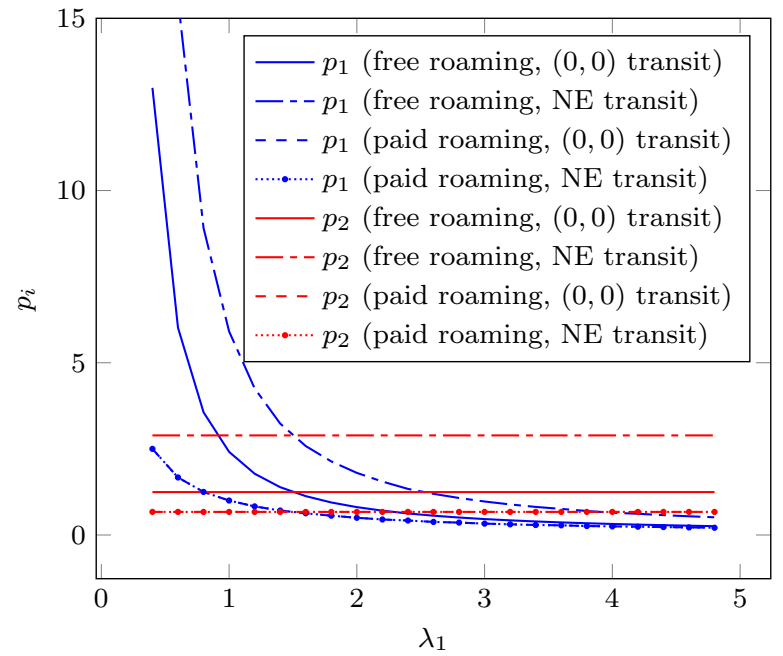

Figure 3: Prices $p_{i}$ in terms of $\lambda_{1}$, for free and paid roaming, and different transit prices. 


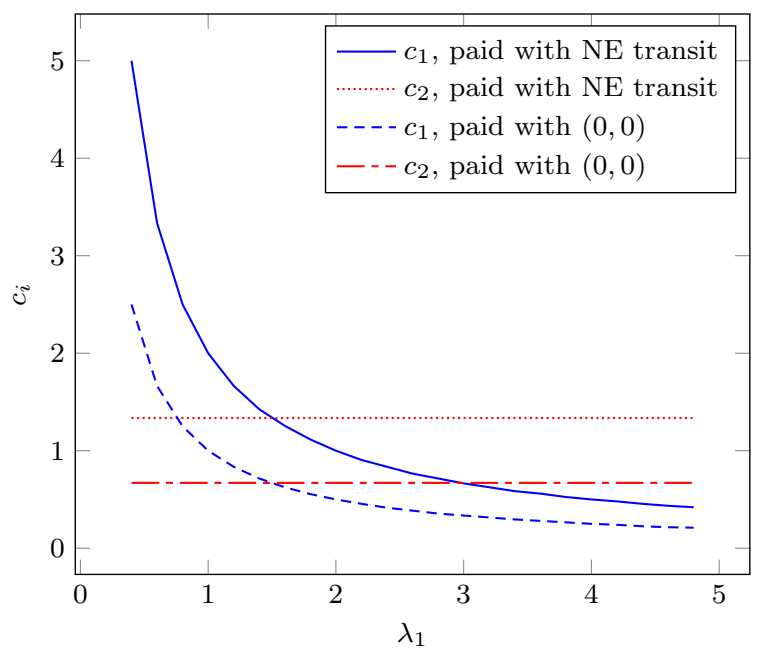

Figure 4: Roaming charges $c_{i}$ in terms of $\lambda_{1}$, for different transit prices.

compensate the transit cost, but quite surprisingly with paid roaming, they are the same; actually, as illustrated on Figure 4, transit prices are compensated by roaming charges.

The level of wealth in a country is illustrated in Figure 5, and as proved earlier, to impact the transit price of the other country (because it can attract more roaming money) but not the domestic transit prices. Interestingly also, there is no strict dominance of transit prices between free and paid roaming: when $\lambda_{1}$ is small, i.e., users are wealthier, the transit price from the other country is larger with paid roaming than with free roaming, but it is the opposite when $\lambda_{1}$ is large; this can probably be related to the increased roaming charge $c_{i}$ which increases with wealth, inducing a possibility to increase the transit prices (indeed, the two values are highly positively correlated).

Revenues in terms of $\lambda_{1}$ are shown in Figure 6. They are decreasing with wealth, which is coherent; only $R_{2}$ is independent of the wealth in Country 1 when transit prices are $(0,0)$, for free or paid roaming. It is also interesting to see that imposing null transit prices may lead to larger revenues for ISPs with respect to prices defined through a game, the preference depending on the level of wealth in the country(ies). But for a fixed strategy for the definition of transit prices, ISP revenues seem always larger with paid roaming than with free roaming.

Looking more closely at the evolution of subscription thresholds $\bar{\theta}_{i}$ in Figure 7 , the wealth distribution in Country 1 still does not impact Country 2, because prices are not impacted there. On the other hand, $\bar{\theta}_{1}$ depends directly on $p_{1}$ (and $c_{1}$ ), hence decreases with $\lambda_{1}$, i.e., when users are less wealthy. For the paid roaming cases, the curves with zero or Nash transit prices are superimposed: there is no dependence on the choice of $\left(t_{1}, t_{2}\right)$.

Figure 8 displays the proportions of subscribers in the different cases; again 


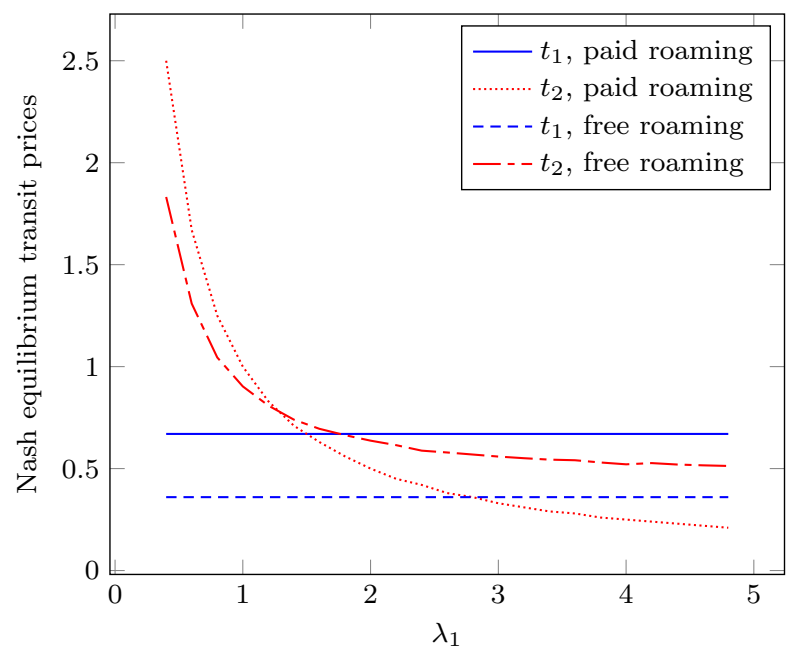

Figure 5: Nash equilibrium transit prices in terms of $\lambda_{1}$

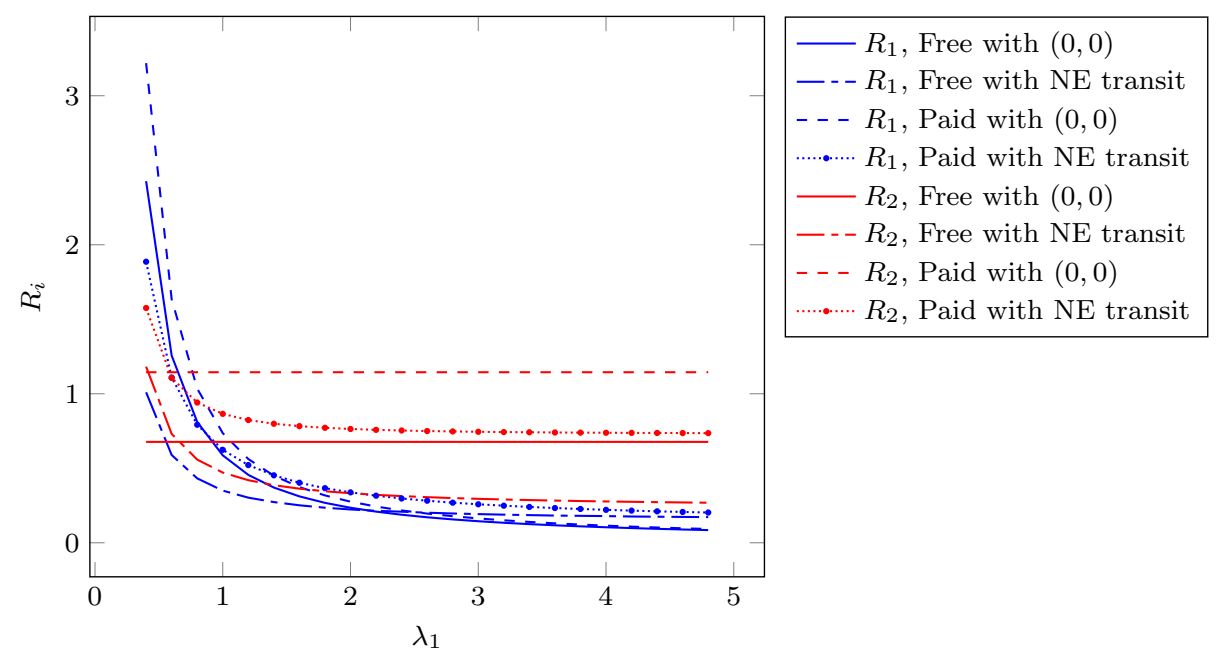

Figure 6: Revenues in terms of $\lambda_{1}$, for free and paid roaming, and different transit prices. 


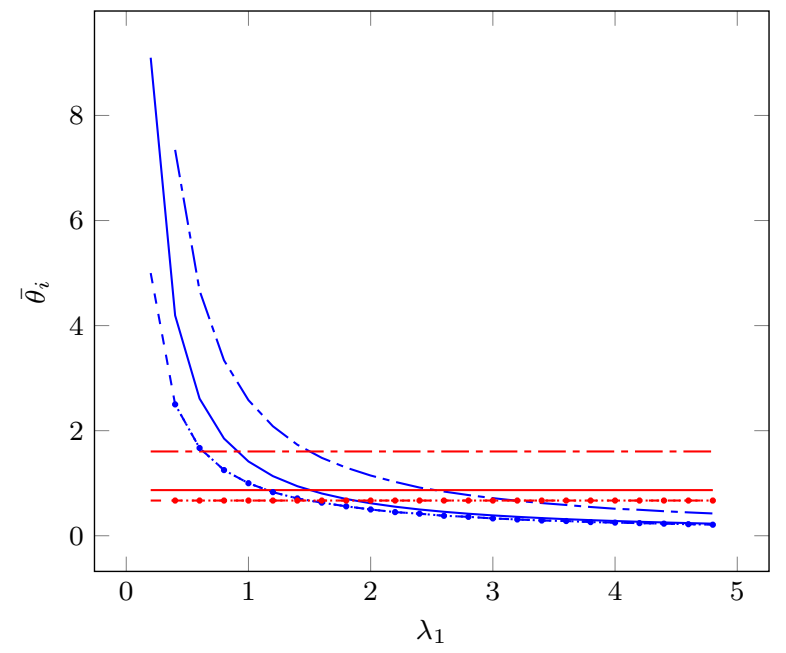

$$
\begin{array}{|l}
-\bar{\theta}_{1}, \text { Free with }(0,0) \\
---\bar{\theta}_{1}, \text { Free with NE transit } \\
---\bar{\theta}_{1}, \text { Paid with }(0,0) \\
\cdots \cdots . \cdots \cdot \bar{\theta}_{1}, \text { Paid with NE transit } \\
-\bar{\theta}_{2}, \text { Free with }(0,0) \\
---\bar{\theta}_{2}, \text { Free with NE transit } \\
---\bar{\theta}_{2}, \text { Paid with }(0,0) \\
\cdots \cdots . . . . . \bar{\theta}_{2}, \text { Paid with NE transit }
\end{array}
$$

Figure 7: Subscription threshold $\bar{\theta}_{i}$ in terms of $\lambda_{1}$

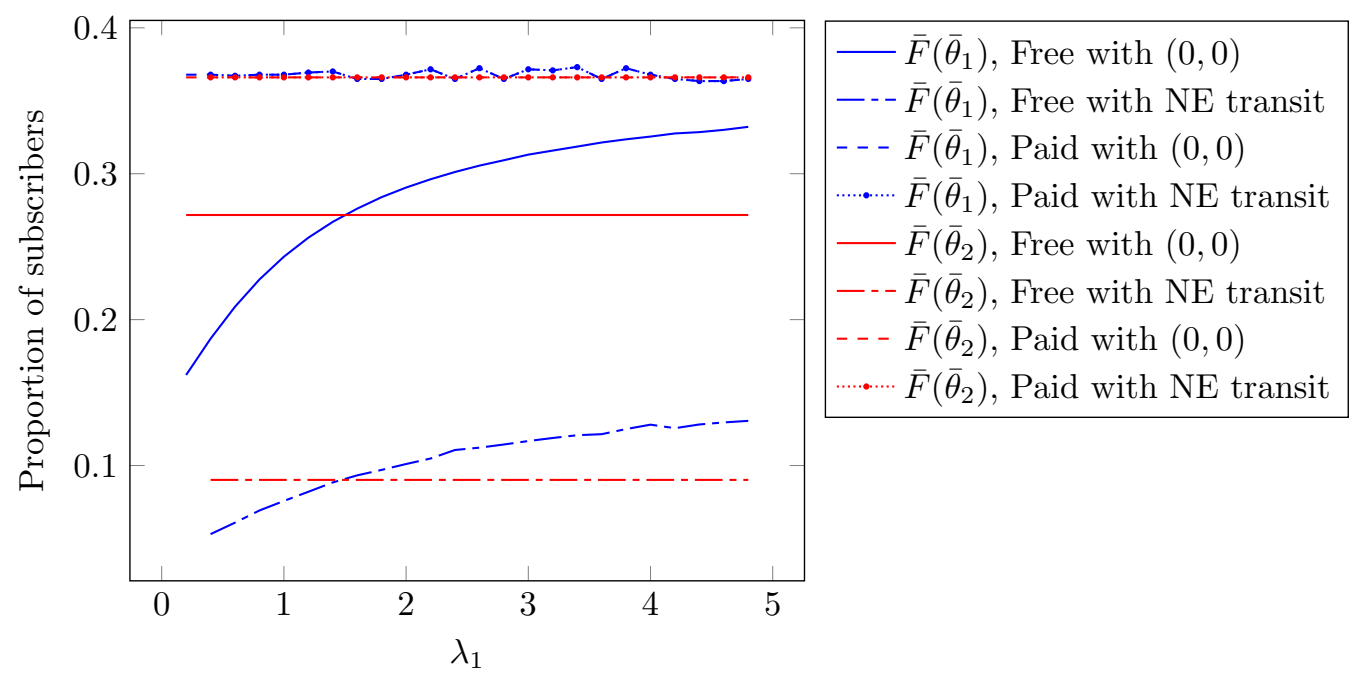

Figure 8: Proportion of users to subscribe in terms of $\lambda_{1}$ 


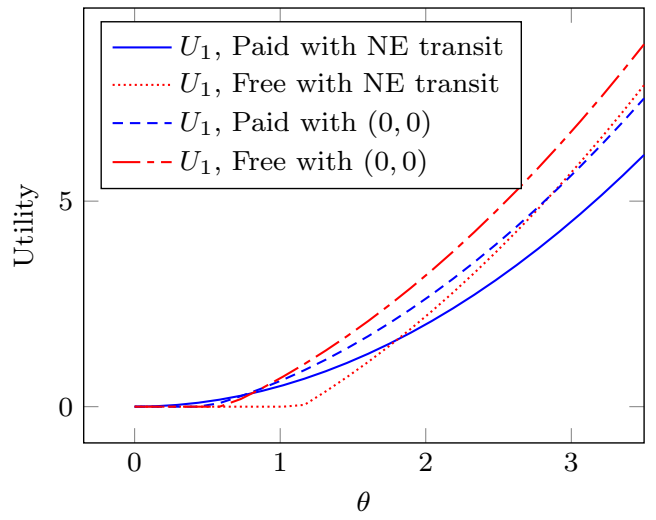

Figure 9: Utility in terms of $\theta$

wealth in a country does not impact subscriptions in the other country. Wealth has no impact on the proportion in the case of paid roaming, which is actually not true for free roaming (ex: the proportion of subscribers in Country 1 in the free and regulated case is increasing as $\lambda_{1}$ increases, because of the price decrease). Paid roaming is actually more interesting if the proportion of subscribers is what we are focusing on.

\section{Users' utility in terms of $\theta$}

We now fix the parameters to $\alpha_{1}=\alpha_{2}=1, m_{1}=1, m_{2}=2, \lambda_{2}=1$ and $\lambda_{1}=2$. We use the prices determined for the four strategies (free or paid roaming, null transit prices or determined through a Nash equilibrium), and display in Figure 9 the individual user utility as a function of $\theta_{1}$.

We can see that for a large $\theta_{1}$ the free options will always be preferred, which is not true when $\theta_{1}$ is small. Hence the consumer surplus should depend on the distribution of wealth, i.e., of $\theta_{1}$. The gap between the two options with transit prices decided through a non-cooperative game shows why the paid option is prefered in this case (given that a small $\theta_{1}$ is much more likely to occur). Also we see why the consumer surplus is larger with the free option with transit prices $(0,0)$.

\section{Conclusions}

We have provided in this paper a model describing the interactions of ISPs in different countries, and subscription choices of users potentially subject to roaming. The goal was to analyze whether or not the decision to impose free roaming in EU in July 2017 is good for users -which is expected- and ISPs -which is less expected-. 
We have illustrated some a priori counter-intuitive results: free roaming is not necessarily better for users than paid roaming (in particular, if transit prices are decided by ISPs through a non-cooperative game), and ISPs' revenues are not necessarily larger if defined by the ISPs themselves. It shows that scrutiny is required before taking a decision.

This work can be extended in several ways. First, we may add competition among ISPs in given countries, or different levels of competition to see if this does bring an asymmetry in the relations. Also some of our results are obtained with full generality while others, even if illustrative of the required scrutiny, are from numerical investigations with specific functions; we would look to generalize those observations.

\section{References}

[1] M. Cho and M. Choi. Pricing for mobile data services considering service evolution and change of user heterogeneity. IEICE Transactions on Communications, E96-B(2):543-552, 2013.

[2] P. Maillé and B. Tuffin. Telecommunication Network Economics: From Theory to Applications. Cambridge University Press, 2014.

[3] P. Maillé and B. Tuffin. Users facing volume-based and flat-rate-based charging schemes at the same time. In IEEE Explore, editor, 8th Latin American Network Operations and Management Symposium (LANOMS 2015), Joao Pessoa, Brazil, October 2015.

[4] P. Maillé and B. Tuffin. Enforcing free roaming among UE countries: an economic analysis. Preprint, https://hal.inria.fr/hal-01428920, 2017.

[5] J. Nash. Two-person cooperative games. Econometrica: Journal of the Econometric Society, pages 128-140, 1953.

[6] M. Osborne and A. Rubinstein. A Course in Game theory. MIT Press, 1994.

[7] T Stühmeier. Roaming and investments in the mobile internet market. Telecommunications Policy, 36:595-607, 2012. 